Finally, the notion of prompt use of a highly precise intercontinental-range missile within an hour's decision time powerfully conveys the longstanding American preference for dabbling with technological solutions to the exclusion of clear-headed strategic thinking. Here, American decision makers would best avoid myopic thinking about the utility of CPGS as a silver bullet. It would be preferable to consider more fully the broad and unwelcome dangers and policy ramifications that could very well result from such narrow thinking.

\title{
Militäreinsätze zwischen menschlicher Sicherheit und wirtschaftlichen Interessen - Perspektive eines evangelischen Ethikers
}

\author{
Volker Stümke*
}

\begin{abstract}
To provide human security is a justified and important political aim. There are different means to achieve it, including, ultimately, the State's monopoly on the use of force. From the perspective of peace ethic, only reluctant use of force is acceptable. The use of military force must remain the last resort (ultima ratio), as politics is both legally and morally bound to peace. Furthermore, securitization itself can overburden the effectiveness of both the state and politics. Political ethics, however, demands that not just political considerations, but also economic, scientific and religious ones, and their implicit acceptance, serve as the linchpins of society. No one aspect should dominate, because that would lead to social imbalance.
\end{abstract}

Military action for economic interests would be an encroachment of politics in economic matters that, although conceivable, is prohibited by law and would be morally compromising. As it has always been wrong to further religious beliefs through military instead of spiritual means, it is similarly both legally and morally wrong to further economic interests with military means instead of trusting economic ones to handle conflict.

Keywords: Ethics, interest, war, military, security, securitization; Ethik, Interesse, Krieg, Militär, Sicherheit, Versicherheitlichung

W ozu darf, sollte, müsste Deutschland Streitkräfte einsetzen? Es gibt seit dem Völkermord in Ruanda eine starke Tendenz zu dem politischen Konsens, dass sich aus menschenrechtlichen Verpflichtungen entsprechende humanitäre Interventionen als Ultima Ratio ableiten und rechtfertigen lassen ${ }^{1}$. Aber gilt das gleichfalls, wenn wirtschaftliche Interessen eines Landes gefährdet sind? Erinnert sei an die umstrittene Äußerung des damaligen Bundespräsidenten Horst Köhler, „dass im Zweifel, im Notfall auch militärischer Einsatz notwendig ist, um unsere

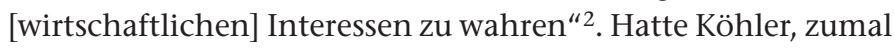
er explizit von einem Notfall gesprochen hat, recht? - Diese

* Prof. Dr. Volker Stümke lehrt evangelische Sozialethik an der Führungsakademie der Bundeswehr in Hamburg und Systematische Theologie an der Kirchlichen Hochschule Wuppertal-Bethel.

Dieser Artikel wurde einem anonymen Begutachtungsverfahren (doubleblind peer-reviewed) unterzogen.

1 Vgl. dazu Bastian Loges, Schutz als neue Norm in den internationalen Beziehungen. Der UN-Sicherheitsrat und die Etablierung der Responsibility to Protect, Wiesbaden 2013.

2 Folgendes Zitat Köhlers aus einem Interview mit dem Deutschlandradio Kultur vom 22. Mai 2010 sorgte für Aufsehen: „Meine Einschätzung ist aber, dass insgesamt wir auf dem Wege sind, doch auch in der Breite der Gesellschaft zu verstehen, dass ein Land unserer Größe mit dieser Außenhandelsorientierung und damit auch Außenhandelsabhängigkeit auch wissen muss, dass im Zweifel, im Notfall auch militärischer Einsatz notwendig ist, um unsere Interessen zu wahren, zum Beispiel freie Handelswege, zum Beispiel ganze regionale Instabilitäten zu verhindern, die mit Sicherheit dann auch auf unsere Chancen zurückschlagen negativ, bei uns durch Handel Arbeitsplätze und Einkommen zu sichern. Alles das soll diskutiert werden, und ich glaube, wir sind auf einem nicht so schlechten Weg." - zitiert nach Frankfurter Rundschau vom 27. Mai 2010.
Fragen sind nicht nur, aber auch für Soldaten relevant, weil sie vom Bundestag und damit im Namen unseres Volkes in solche Einsätze gerufen werden ${ }^{3}$. Indem dies eben in unserem Namen als Staatsbürger geschieht, stehen auch wir vor den Fragen, ob solche Einsätze rechtlich legal und ethisch legitim wären.

Ich möchte zu diesen Fragen aus der Perspektive eines evangelischen Ethikers Stellung beziehen. Dabei werden zunächst die beiden im Titel vorgegebenen Begriffe „wirtschaftliche Interessen“ und „menschliche Sicherheit“ präzisiert, denn viel hängt davon $a b$, dass die Kontroversen mit der nötigen wissenschaftlichen Klarheit in den Blick genommen werden. Die Ethik als Wissenschaft hat Niklas Luhmann folgend „die vielleicht vordringlichste Aufgabe [...], vor Moral zu warnen“4. Sofern damit gemeint ist, Vorbehalte gegenüber einer ungezügelten und assoziativen Verwendung moralischer Gefühle und gegenüber einem vermeintlichen Wertekonsens, der durch plakative Beispiele herbeigerufen wird, zu formulieren, ist dem zuzustimmen. Nach der also durchaus moralwissenschaftlich ${ }^{5}$

3 Vgl. Hartwig von Schubert, Die Ethik rechtserhaltender Gewalt, Opladen 2013 4 Vgl. Niklas Luhmann, Paradigm lost. Über die ethische Reflexion der Moral, Frankfurt/Main 1990, 40f.

5 Anders als die Frankfurter Schule (von Adorno über Habermas bis zu Forst) verwende ich in Übereinstimmung mit den meisten evangelischen Ethikern die Begriffe Ethik und Moralphilosophie synonym. Gemeint ist die Lehre von der Moral (Personalethik) und von den Sitten (Sozialethik). Es wird also davon ausgegangen, dass nicht nur die gesellschaftlichen, sondern auch die persönlichen Werte und Normen wissenschaftlich reflektiert und mit guten Gründen beurteilt werden können. 
relevanten Präzision der Begriffe werde ich im dritten Schritt auf Argumente aus der evangelischen Theologie zurückgreifen, um zu einer Antwort zu gelangen.

\section{Wirtschaftliche Interessen}

Begriffsgeschichtlich ist der Ausdruck „Interesse“ mit einer Vielzahl von Konzeptionen verbunden ${ }^{6}$. Er gehört zunächst in den Kontext des römischen Rechts und meint den Schadensersatz (und später den Zins). Im Mittelalter wird das verallgemeinert, Interesse bezeichnet nun das, was Nutzen stiftet. Das kann einerseits individuell verstanden werden und meint dann den Eigennutz, was vor allem in der christlichen Morallehre pejorativ aufgefasst wurde: Das Interesse des Sünders kreist nur um sich selbst, er ist in sich selbst verkrümmt (Martin Luther ${ }^{7}$ ). In der Aufklärung wurde diese Bewertung zurückgenommen, die „Bedürfnisse“ der Menschen sind nunmehr ethisch neutral oder dienen sogar dem wirtschaftlichen Fortschritt (Adam $S_{\text {Smith }}{ }^{8}$ ). Andererseits entwickelte sich eine politische Interpretation, die das Interesse mit dem Gemeinwohl des Staates verband (Thomas Morus ${ }^{9}$ ). Schließlich gab es eine psychische und später ästhetische Modifikation, nunmehr geht es um eine innere Beteiligung, die durch einen Kunstgegenstand evoziert wird, der „interessant“ ist. In der Erkenntnistheorie wurde der Vernunft ein spekulativer Hang unterstellt, die Welt systematisch zu erfassen (Immanuel Kant ${ }^{10}$ ); inzwischen wurde dieses Interesse in unterschiedliche erkenntnisleitende Fragestellungen ausdifferenziert (Jürgen Habermas ${ }^{11}$ ). Der Begriff "Interesse" hat also eine rechtliche, eine personalethische, eine politische, eine ästhetische und eine erkenntnistheoretische Extension. Aber immer bezeichnet er das Ausgerichtetsein eines Menschen - ebenso wie die Begriffe "Affekt" und „Wunsch“. Im Unterschied zu einem Affekt ist das Interesse allerdings reflektiert und lässt sich mit Gründen plausibilieren, der (inhaltlich durchaus divergente) Bezug zur Vernunft gehört also konstitutiv zum Interesse, ebenso wie die Relation zum Subjekt und das weitgehend aktive Moment dieses Subjekts. Im Unterschied zu einem Wunsch, der durchaus utopische Ziele in den Blick nehmen kann, ist ein Interesse stärker an der (allerdings gestaltbaren, also nicht statisch vorgegebenen) Wirklichkeit orientiert.

Dass Menschen wie Institutionen bis hin zu Staaten Interessen haben, wird in ethischer Perspektive weitgehend als Sachverhalt akzeptiert, der noch nicht bewertet wird. Interessen an sich

6 Vgl. zum Folgenden: Hans-Jürgen Fuchs, Art.: Interesse I (bedeutungsgeschichtlich); in: Historisches Wörterbuch der Philosophie Band 4, hrsg. von Joachim Ritter und Karlfried Gründer, Darmstadt 1976, 479-485 und Volker Gerhardt, Art.: Interesse II (philosophisch); in: Historisches Wörterbuch der Philosophie Band 4, hrsg. von Joachim Ritter und Karlfried Gründer, Darmstadt 1976, 485-494.

7 Vgl. Martin Luther, Römerbriefvorlesung zu Röm 8,3 von 1515/16 (WA 56, 356, 5f.: „homo incurvatus in se“).

8 Vgl. dazu Claudius Luterbacher-Maineri, Adam Smith - Theologische Grundannahmen. Eine textkritische Studie, Fribourg 2008, 328-364.

9 Vgl. Thomas Morus, Utopia. Zweites Buch (1516), Stuttgart 2003, vor allem 58-66.

10 Vgl. Immanuel Kant, Kritik der reinen Vernunft, 2. Auflage Riga 1787, 670ff. (Werke in zehn Bänden, hrsg. von Wilhelm Weischedel, 5. Auflage Darmstadt 1983, Band IV, 563ff.)

11 Vgl. Jürgen Habermas, Erkenntnis und Interesse, Frankfurt/Main 1973, 234ff. und ders., Theorie des kommunikativen Handelns. Band 1: Handlungsrationalität und gesellschaftliche Rationalisierung, 4. Auflage Frankfurt/Main 1987, 15-196. sind also weder gut noch schlecht - und das gilt für persönliche Vorlieben wie für gesellschaftliche Ziele, aber es gilt weder für jeden Wunsch noch für jeglichen Affekt ${ }^{12}$. Moralphilosophisch weitaus relevanter ist der zweite Schritt, nämlich die Umsetzung des Interesses in entsprechende Handlungen. Denn es kann zu Interessenskollisionen kommen, sei es, weil mehrere Personen dasselbe Interesse haben und nun in Konkurrenz zueinander stehen, sei es, dass umgekehrt in einer Gruppe divergierende Interessen favorisiert werden und daher eine Priorisierung erforderlich wird. Nunmehr müssen sowohl die Mittel ethisch geprüft, als auch die Folgen des Handelns kalkuliert und bewertet werden. Weniger die Interessen, wohl aber die Wege, sie zu realisieren, sind also ethisch zu analysieren.

Das gilt gleichermaßen für wirtschaftliche Interessen. Auch sie sind ethisch zunächst neutral, aber mit welchen Mitteln sie umgesetzt und welche Folgen sie zeitigen werden, ist ethisch relevant. Allerdings kommt eine neue Schwierigkeit hinzu, die schon grammatisch angezeigt wird. Der Genitiv „Interessen der Wirtschaft" kann sowohl objektiv wie subjektiv gemeint sein. Im ersten Fall wird ausgesagt, dass wir Interesse an der Wirtschaft haben, dass wir ein funktionierendes Wirtschaftssystem wollen oder dass wir bereit sind, uns für wirtschaftliche Belange zu engagieren. Mit dem subjektiven Genitiv wird nahe gelegt, dass die Wirtschaft, obwohl sie ein Abstraktum ist, dennoch eigene Interessen habe. So wollen Unternehmer kurzfristig Gewinn machen und sich nachhaltig am Markt behaupten. Sicherlich sind beide Lesarten berechtigt, sie ergänzen sich. Allerdings insinuieren das „wir“ wie der Kollektivsingular „Wirtschaft" eine Einheit, die im Paradigma der Ökonomie nicht in der Realität, sondern erst auf einer Reflexionsebene anzusiedeln ist.

Es gibt also zwei Ebenen bei der Rede von wirtschaftlichen Interessen. Auf der ersten Ebene geht es um den Bezug zur Realität. Die Ökonomik geht weitgehend davon aus, dass es faktisch Knappheiten an Gütern und Faktoren (Arbeit, Kapital, Boden - vielleicht auch Know-how) gibt, deren Allokation am effizientesten im freien Handel auf dem Markt geregelt werden sollte ${ }^{13}$. Hier gibt es keine einheitlichen wirtschaftlichen Interessen, sondern Konkurrenz zwischen den handelnden Akteuren. Wer nach einer Einheit sucht, wird auf die zweite, höhere Ebene verwiesen, namentlich das Miteinander der Menschen in einer Polis, die insgesamt nur funktioniert, wenn auch der Bereich der wirtschaftlichen Interaktionen (Handel, Arbeitsleistung, Dienstleistung) in ihr angemessen abgebildet wird. Auf dieser zweiten Ebene gibt es ein gemeinsames Interesse der unterschiedlichen wirtschaftlichen Akteure beispielsweise an einem freien Handel und an freien Handelswegen - aber dieses Interesse steht nun neben den Interessen anderer Akteure in der Polis, also beispielsweise den Interessen der Politiker, anderer gesellschaftlicher Gruppen und nicht zuletzt den In-

12 Gegenüber der umgangssprachlichen Behauptung, man habe bspw. ein Interesse daran, jemanden zu töten, wird hier behauptet, dass eine solche Tat entweder ein Affekt sei oder aber ein (unangemessenes) Mittel, um ein Interesse (bspw. an einem Gegenstand, den der andere besitzt) durchzusetzen. Das Interesse selbst kann nämlich auch auf anderem Weg (bspw. durch Kauf) umgesetzt werden und der wäre ethisch nicht zu beanstanden. Auch die Rede, dass man ein Interesse hätte, in andere Galaxien zu reisen, wäre gemäß meiner Begriffsdefinition kein Interesse, sondern ein - zumindest derzeit - utopischer Wunsch, der aber vielleicht einmal zu einem realen Interesse werden kann.

13 Vgl. zum Folgenden Karl Homann und Andreas Suchanek, Ökonomik. Eine Einführung, 2. überarbeitete Auflage, Tübingen 2005. 
teressen der einzelnen Bürger. Und nunmehr kann es zwischen diesen Interessen der unterschiedlichen politischen Akteure zu Divergenzen kommen.

Mit dem evangelischen Ethiker Eilert Herms lassen sich auf der zweiten Ebene vier Säulen unterscheiden, die allesamt das Zusammenleben in einer Polis tragen: Politik, Wirtschaft, Wissenschaft und persönliche Sinndimension (bspw. Religion) ${ }^{14}$. Jeder Säule komme dabei eine relative Eigenständigkeit zu. Es gebe also jeweils bestimmte Aufgaben und Ziele (Frieden, Lebensunterhalt, Naturbeherrschung, Sinn), die erreicht werden sollen, und spezifische Mittel, die der jeweiligen Zielverfolgung dienlich seien (Recht, Markt, Forschung, Herzensbildung). Ihr Miteinander müsse immer wieder austariert werden, und immer führten sowohl die Dominanz wie auch das Wegbrechen einer Säule zu einer gesellschaftlichen Schieflage. Mit Blick auf die wirtschaftlichen Interessen sind also weder ihre Vernachlässigung noch ihre Überbetonung aus ethischer Perspektive geboten.

Dass den Säulen eine relative Eigenständigkeit zukommen soll, schließt wechselseitige Einwirkungen ein. So haben sowohl politische wie wissenschaftliche und religiöse Handlungen auch wirtschaftliche Effekte. Der Ausbau von Kindergartenplätzen, der Kampf gegen Krebs und auch die persönliche Zufriedenheit wirken sich auf den Markt aus - aber das sind noch keine wirtschaftlichen Interessen, sondern eben gleichsam kollaterale Nebenwirkungen. Auch wenn es in der Realität bisweilen schwierig ist, solche Effekte von Interessen klar zu unterscheiden, so müssen sie doch begrifflich differenziert werden - nur so kann die analytische Dominanz eines Themenbereichs (bzw. einer Säule) mit den entsprechenden ausufernden Deutungen bis hin zu Verschwörungstheorien ausgeschlossen werden ${ }^{15}$. Daher werden im Folgenden nicht die Effekte, sondern die Interessen im Fokus der Analyse stehen.

Für die ethische Analyse wirtschaftlicher Interessen gilt demzufolge, dass sie nicht nur (gleichsam betriebswirtschaftlich) auf die Mittel und die Folgen wirtschaftlichen Handelns fokussiert sein darf, sondern zudem das Verhältnis des ökonomischen Denkens zu den anderen Größen in einer Polis (volkswirtschaftlich) zu evaluieren hat. Und jetzt gerät die Politik in den Fokus, weil die wirtschaftlichen Interessen der zweiten Ebene innerhalb der Polis verhandelt und bestimmt werden. Hier geht es darum, ob und mit welchen Mitteln man wirtschaftliche Interessen politisch unterstützen darf und soll. Sie hat dabei nicht nur zu beachten, inwiefern ihre Maßnahmen im internen Konkurrenzkampf auf der ersten Ebene bestimmte Parteien unterstützen oder belasten ${ }^{16}$. Sie muss zudem auf der

14 Vgl. Eilert Herms, Grundzüge eines theologischen Begriffs sozialer Ordnung; in: ders., Gesellschaft gestalten. Beiträge zur evangelischen Sozialethik, Tübingen 1991, 56-94. Näherhin würden Politik und Wirtschaft das Miteinander von Personen als Sicherung des Zusammenlebens und des Lebensunterhalts regeln, während die beiden anderen auf individuelle Handlungsgewissheit im technischen und im ethischen Bereich zielten.

15 Damit wird behauptet, dass die Pointe solcher Verschwörungstheorien darin liegt, vorab schon von der Dominanz eines Themenbereichs oder eines Akteurs überzeugt zu sein - und dies dann in jeglichem Handeln als Effekt zu entdecken, der nunmehr zum Interesse aufgeplustert wird.

16 So sind beispielsweise Subventionen bestimmter Wirtschaftsbranchen (wie Kohleabbau) zugleich eine Benachteiligung konkurrierender Unternehmer auf der ersten Ebene (der konkurrierenden Energieversorger), die aber auf der zweiten Ebene durch politische Ziele (wie Arbeitsplatzsicherung oder Energieautarkie) gleichsam übersteuert werden können. zweiten Ebene bedenken, dass ihre Beförderung wirtschaftlicher Interessen zu Lasten anderer gesellschaftlicher Bereiche oder Kräfte gereichen $\mathrm{kann}^{17}$ - und weil die Politik sowohl nach innen wie nach außen dem Frieden als ihrem Ziel verpflichtet ist $^{18}$, sollte sie solche Belastungen sorgsam prüfen. Inzwischen gibt es zudem noch drittens die globale Ebene, weil viele Wirtschaftsunternehmen international tätig sind ${ }^{19}$.

Dass die Politik nunmehr ins Blickfeld geraten ist, soll aber nicht besagen, dass sie der einzige Handlungsträger sei. Es gibt diesbezüglich unterschiedliche wirtschaftsethische Konzepte, die hier nur angedeutet, aber nicht eigens dargestellt noch gewürdigt werden sollen ${ }^{20}$. Manche betonen den Staat als maßgebliche Instanz, welche die Rahmenbedingungen für die Wirtschaft schaffen muss (Karl Homann ${ }^{21}$ ), andere hingegen setzen vorrangig auf Unternehmen, die sich selbst (und wechselseitig) auf bestimmte Standards verpflichten (Josef Wieland ${ }^{22}$ ), wieder andere nehmen die Konsumenten als mündige Bürger in die Pflicht, ihr Marktverhalten bewusst zu gestalten und das politisch umzusetzen (Peter Ulrich ${ }^{23}$ ), und schließlich gibt es Konzepte, die auf die intrinsische Motivation setzen (Bruno Frey ${ }^{24}$ ), um konkrete Veränderungen zu erwirken. Die Politik rückt deshalb in den Vordergrund, weil im Hintergrund die Frage nach der militärischen Gewaltanwendung lauert - und sie ist eindeutig im Bereich der Politik zu verorten.

\section{Menschliche Sicherheit}

Dieser hintergründige Bezug auf Militär und Politik wird durch den Begriff der Sicherheit im Titel angezeigt. Klassisch meinte dieser Begriff im politischen Kontext den Schutz der Bürger durch den Staat und durch seine Exekutive ${ }^{25}$. Während die Polizei die innere Sicherheit gewährleisten soll, indem sie Verbrechen bekämpft, wird das Militär benötigt, um die äußere Sicherheit als Schutz vor dem Angriff durch andere Länder herzustellen. Allerdings ist dieses Verständnis seit dem Ende des Kalten Krieges mehrfach aufgebrochen und der Begriff

17 So wird beispielsweise die Ausweitung der Ladenöffnungszeiten, die aus wirtschaftlicher Sicht zumeist befürwortet wird, von anderen gesellschaftlichen Gruppen wie Sportvereinen, Kirchen und Gewerkschaften kritisiert, weil sie auf der zweiten Ebene gleichsam Benachteiligungen fürchten. Aber es mag auch auf der ersten Ebene Unterschiede geben, sofern die verlängerten Arbeitszeiten und die damit verbundenen Kosten nicht für alle Unternehmen lukrativ sein können.

18 Diese Friedensnorm der Politik gilt sowohl theoretisch (vgl. dazu Dolf Sternberger, Begriff des Politischen [Heidelberger Antrittsvorlesung]; in: ders., Die Politik und der Friede, Frankfurt/Main 1986, 69-88) wie in Deutschland faktisch (vgl. die Präambel des Grundgesetzes: „dem Frieden der Welt zu dienen").

19 Dass die Globalisierung erhebliche Auswirkungen für sowohl Wirtschaftsunternehmen wie Politik hat, steht außer Frage. Vgl. dazu grundlegend Christoph Scherrer und Caren Kunze, Globalisierung, Göttingen 2011. An dieser Stelle können aber diese Modifikationen nicht vertieft werden.

20 Vgl. zum Folgenden das Handbuch der Wirtschaftsethik Band 1.2: Verhältnisbestimmung von Wirtschaft und Ethik, hrsg. von Wilhelm Korff, Berlin 2009, 683-780 und 834-883.

21 Vgl. Karl Homann und Franz Blome-Drees, Wirtschafts- und Unternehmensethik, Göttingen 1992 und Christoph Lütge und Karl Homann, Einführung in die Wirtschaftsethik, Berlin 2013.

22 Vgl. Josef Wieland, Die Ethik der Governance, 5. Auflage Weimar 2007 und ders., Die moralische Verantwortung kollektiver Akteure, Wiesbaden 2013.

23 Vgl. Peter Ulrich, Integrative Wirtschaftsethik. Grundlagen einer lebensdienlichen Ökonomie, Bern 1997 und ders., Zivilisierte Marktwirtschaft. Eine wirtschaftsethische Orientierung, Bern 2005.

24 Vgl. Bruno S. Frey, Markt und Motivation. Wie ökonomische Anreize die (Arbeits-) Moral verdrängen, München 1997.

25 Vgl. Harald Kleinschmidt, Legitimität, Frieden, Völkerrecht. Eine Begriffsund Theoriegeschichte der menschlichen Sicherheit, Berlin 2010. 
der Sicherheit dementsprechend aufgefächert worden. Sehr klar hat Christopher Daase diese Erweiterungen dargelegt ${ }^{26}$. Er unterscheidet vier Dimensionen:

1. Raumdimension: Der Bereich der Sicherheit hat sich vergrößert, indem nicht mehr nur die Nation, sondern zunächst die Region und inzwischen die eine globale Welt der Ort ist, der sicher sein soll.

2. Sachdimension: Als Bedrohung der Sicherheit sind neben den militärischen Kräften nunmehr auch ökonomische, ökologische und humanitäre Katastrophen in das Blickfeld gelangt. Aus wirtschaftlichen Interessen kann demnach eine Bedrohung der Sicherheit erwachsen: Wenn in einem Staat die Versorgung mit Grundgütern nicht mehr gewährleistet ist, dann könnten ein Bürgerkrieg oder ein Angriffskrieg drohen, um sich die Güter mit Gewalt zu verschaffen. Aber es könnte auch zu starken Migrationsbewegungen kommen, welche die aufnehmenden Nachbarländer vor Probleme stellten.

3. Gefahrendimension: Als Gefährdung der Sicherheit gilt nicht erst ein Angriff oder eine Bedrohung, sondern werden inzwischen auch schon die Verwundbarkeit und das Risiko angesehen.

4. Referenzdimension: Geschützt werden soll nicht mehr nur der Staat, sondern auch die Gesellschaft und sogar der Einzelne.

Dieser Ausdifferenzierung korrespondiert, dass Staat und Militär nicht mehr allein die Sicherheit gewährleisten können. Die Bundeswehr kann unser Land als Raum verteidigen, aber kann sie auch die ganze Welt retten? Sie kann der Sache nach einen Angriffskrieg abwehren, doch eine Bankenpleite, das Abschmelzen der Pole und eine Epidemie reagieren nicht auf Waffengewalt. Eine faktische militärische Bedrohung mag man durch ein Gleichgewicht des Schreckens ausbalancieren, aber zukünftige Risiken kann man nur dann einhegen, wenn man sie präzise kennt - und dann sind es eben keine Risiken mehr, sondern Bedrohungen. Und noch nie hat es das Militär vermocht, wirklich jeden Einzelnen zu beschützen - aber genau das besagt die individualisierte Referenzdimension. Aus der Differenzierung der Sicherheit folgt also eine Reduktion und zugleich Präzision des Aufgabenspektrums der Bundeswehr. Dementsprechend wird dort schon seit Jahren eine erweiterte und vernetzte Sicherheitskonzeption politisch gefordert und militärisch entwickelt, in die andere Akteure neben der Bundeswehr einbezogen werden ${ }^{27}$.

Der Begriff der menschlichen Sicherheit gehört in diesen Kontext ${ }^{28}$. Er wurde in den letzten 20 Jahren zunächst im Entwicklungsprogramm der Vereinten Nationen (UNDP) publik gemacht und daraufhin im Kontext der „Entwicklungshilfe“ profiliert und besagt, dass zur Schutzbedürftigkeit des Menschen (und eben nicht mehr zur Sicherheit des Staates) grundlegend

26 Vgl. zum Folgenden Christopher Daase, Von der nationalen zur menschlichen Sicherheit. Politische und rechtliche Konsequenzen des erweiterten Sicherheitsbegriffs; in: Recht und Politik globaler Sicherheit. Bestandsaufnahme und Erklärungsansätze, hrsg. von Andreas Fischer-Lescano und Peter Mayer, Frankfurt/Main 2013, 11-42.

27 Vgl. Vernetzte Sicherheit. Leitidee der Sicherheitspolitik im 21. Jahrhundert, hrsg. von Heiko Borchert, Hamburg 2004.

28 Vgl. zum Folgenden Cornelia Ulbert und Sascha Werthes, Menschliche Sicherheit - Der Stein der Weisen für globale und regionale Verantwortung; in: Menschliche Sicherheit. Globale Herausforderungen und regionale Perspektiven, hrsg. von dens., Baden-Baden 2008, 13-27. die Freiheit von Angst (freedom from fear = enge, kanadische Lesart) und die Freiheit von Not (freedom from want = weite, japanische Lesart) zählen ${ }^{29}$. Inzwischen werden teilweise auch die durch die Menschenrechte inhaltlich gefüllte Rechtssicherheit (the right to have rights) und die auf Bildung zielende Befähigung, für die eigenen Belange einzutreten (empowerment), hinzugezählt; sie markieren weitgehend die positive Seite der beiden negativen Freiheiten. Entscheidend sind jedenfalls zwei Aspekte: zum einen der durchaus emanzipatorische Bezug auf den Einzelnen und zum anderen die sich dem immer stärker anpassende Profilierung der Sicherheit ${ }^{30}$. Dass damit der Mensch an sich und nicht erst als Staatsbürger in den Fokus auch der klassisch staatszentrierten Sicherheitspolitik gerät, ist aus ethischer Perspektive als Fortschritt zu würdigen. Zugleich trägt auch die Rede von der menschlichen Sicherheit dazu bei, dass nicht mehr Staat und Militär als die einzigen Adressaten dieser Forderungen gelten können. Sie sind nicht einmal mehr die primären Ansprechpartner, denn es geht darum, möglichst allen Menschen Entfaltungsmöglichkeiten zu eröffnen, indem entsprechende Hindernisse identifiziert und abgebaut werden - und zwar vor allem durch zivile Organisationen und gesellschaftliche Institutionen.

Schließlich gibt es neben der Auffächerung des Sicherheitsverständnisses und insbesondere der Individualisierung des Bedarfsträgers noch eine weitere Modifikation, die allerdings zumeist nur am Rande bedacht wird. Auch der Begriff „menschliche Sicherheit" ist subjektiv wie objektiv lesbar. Auf dem objektiven Schutz der Menschen vor Gefahren liegt derzeit das Schwergewicht, während der subjektiven Gewissheit von Menschen wenig Beachtung geschenkt wird. Es geht also überwiegend darum, die objektiven Faktoren, welche Angst verursachen, zurückzudrängen, jedoch kaum darum, subjektive Überzeugungen oder intersubjektive Vernetzungen, welche Vertrauen gegen die Angst aufbauen, zu unterstützen ${ }^{31}$. Sicherlich ist es nicht die Aufgabe des Staates in unserer pluralen und säkularen Gesellschaft, Sinnfragen zu beantworten - dafür gibt es andere Säulen, insbesondere die Religionen ${ }^{32}$. Aber augenscheinlich können sie, anders als die Wirtschaft, ihre Antwortoptionen derzeit bei uns nicht sonderlich erfolgreich vermitteln. Je-

29 Vgl. Tobias Debiel und Sascha Werthes, Menschliche Sicherheit: Fallstricke eines wirkungsmächtigen Konzepts; in: Verunsicherte Gesellschaft - überforderter Staat. Zum Wandel der Sicherheitskultur, hrsg. von Christopher Daase et al., Frankfurt/Main 2013, 319-336.

30 Vgl. Lothar Brock, Human Security zwischen staatlicher Instrumentalisierung und Weltgesellschaftspolitik. Ein Kommentar; in: Verunsicherte Gesellschaft - überforderter Staat. Zum Wandel der Sicherheitskultur, hrsg. von Christopher Daase et al., Frankfurt/Main 2013, 371-388.

31 Vgl. Johano Strasser, Gesellschaft in Angst. Zwischen Sicherheitswahn und Freiheit, Gütersloh 2013, 171: „In normalen Zeiten kann die Mehrheit der Menschen sich mit einer gewissen Gelassenheit damit abfinden, auf die letzten Fragen keine schlüssigen Antworten zu haben, kann sich milde Skepsis im Theoretischen mit Zugewandtheit und Vertrauen in der sozialen Praxis zu einem tragfähigen Lebenskonzept verbinden. In Umbruchzeiten, wie wir sie heute erleben, in Zeiten, da das traditionelle wissenschaftlich-technischökonomische Fortschrittsmodell, das so lange sinn- und sicherheitsstiftend wirkte, an Grenzen stößt und eine allgemeine Zukunftsangst um sich greift, ist das für die meisten Menschen offenbar nicht genug“.

32 Indem die Frage nach dem Sinn des Lebens der Religion zugeordnet wird, wird natürlich nicht behauptet, dass die Religion der Politik oder der Wirtschaft zuarbeitet. Wohl aber wird betont, dass, indem die Religion auf diese Fragen Antworten formuliert, sie dem Menschen Gewissheiten vermitteln und damit Kontingenzerfahrungen, aber auch Erfahrungen von Ohnmacht und Schuld ebenso wie Freude und Dankbarkeit bewältigen kann. Dass solche religiösen Gewissheiten einen gesellschaftlichen Konsens sowohl bestärken wie hinterfragen können, also durchaus riskant sind, betont mit Recht Rolf Schieder, Sind Religionen gefährlich? Berlin 2008 
denfalls führt die stärkere Gewichtung der objektiven Lesart dazu, dass der Staat als Adressat wenig entlastet wird durch diejenigen Institutionen der Religion oder der Sinnstiftung, die das subjektive Pendant der Sicherheit profilieren könnten.

Die gestiegenen Anforderungen an die zu erbringende Sicherheit führen derzeit den Staat und seine Exekutive an ihre Leistungsgrenze $^{33}$. So sehr aus ethischer Perspektive die Einbeziehung des Einzelnen in den Kontext von Politik und Völkerrecht zu begrüßen und so erfreulich es ist, dass die Menschenrechte politisch an Relevanz gewinnen, so problematisch ist es, wenn die entsprechenden politischen Akteure überfordert und verunsichert sind. Wenn einerseits die Sicherheitsmaßnahmen, die der Staat ergreifen kann, begrenzt sind, andererseits aber die Ansprüche steigen, dann kommt es - wie in der Wirtschaft - zu einem Konkurrenzkampf um die knapper werdende Ressource Sicherheit. Dieses Phänomen führt Ole Wæver, dem führenden Vertreter der Kopenhagener Schule, folgend zur Versicherheitlichung (securitization): Demnach werde von unterschiedlichen Trägern dafür geworben, dass die von ihnen beobachteten und als gravierend eingeschätzten Entwicklungen als Risiken zu bewerten und damit sicherheitspolitisch relevant und vom Staat zu bearbeiten seien ${ }^{34}$. Folglich kann eine Vogelgrippe ${ }^{35}$, ein Reaktorunfall in Japan, ein Terrorakt ${ }^{36}$ oder auch die Piraterie am Horn von Afrika als Gefährdung der menschlichen Sicherheit auf die Agenda der Politik geraten - auch wenn sie im klassischen Sinn den Staat kaum oder zumindest nur indirekt gefährden. Weil aber inzwischen alles potenziell sicherheitsrelevant ist, nicht alles jedoch gleichermaßen Berücksichtigung finden kann, findet eine Versicherheitlichung statt, durch die viele Themen in den Genuss der politischen Aufmerksamkeit gelangen ${ }^{37}$.

Als Zwischenergebnis beider Kapitel möchte ich festhalten, dass sowohl wirtschaftliche Interessen wie menschliche Sicherheit aktuelle Herausforderungen für die Politik und damit auch für eine politische Ethik darstellen. Damit steht die Frage im Raum, ob und wann die militärische Gewalt als klassischer Funktionsträger für Sicherheit auch für die Gewährleistung dieser beiden Sicherheitsdimensionen eingesetzt werden darf. Zum einen stellt sich also die Frage, ob menschliche Sicherheit durch Militäreinsätze gesichert oder wiederhergestellt werden darf. Gibt es eine politische Schutzverantwortung (responsibility to protect), die in bestimmten Fällen sogar durch das Mittel des Militärs gewährleistet werden darf? Bei der Beantwortung dieser Frage bildet sich derzeit wohl ein Konsens heraus, dass es eine solche Schutzverantwortung geben sollte und dass militärische Gewalt - allerdings nur als Ultima Ratio und nur in präzise bestimmten Ausnahmefällen - hierzu einen Beitrag

33 Vgl. Christopher Daase und Stefan Engert und Julian Junk, Gesellschaftliche Verunsicherung als Herausforderung des Staates: Eine Einführung; in: Verunsicherte Gesellschaft - überforderter Staat. Zum Wandel der Sicherheitskultur, hrsg. von dens., Frankfurt/Main 2013, 9-32.

34 Vgl. Barry Buzan und Ole Wæver und Jaap de Wilde, Security: A New Framework for Analysis, Boulder 1998.

35 Vgl. Tine Hanrieder und Christian Kreuder-Sonnen, Souverän durch die Krise: Überforderte Staaten und die (Selbst-) Ermächtigung der WHO; in: Verunsicherte Gesellschaft - überforderter Staat. Zum Wandel der Sicherheitskultur, hrsg. von Christopher Daase et al., Frankfurt/Main 2013, 169-186.

36 Vgl. Peter Waldmann, Sicherheit versus Sicherheitsgefühl: Die politischen Auswirkungen des Terrorismus; in: Sicherheit, hrsg. von Nikolaus Werz, Baden-Baden 2009, 17-29.

37 Vgl. Sabine Jaberg, Das Mantra Sicherheit. Reflexionen zu seiner immanenten Logik, HSW kontrovers 6, Hamburg 2012. leisten könne und dürfe ${ }^{38}$. Daher werde ich diese Frage hier nicht weiter verfolgen. Zum anderen kann man fragen, ob die Gefährdung wirtschaftlicher Interessen dazu führen kann und darf, auf das Militär und seine Gewaltanwendung zurückzugreifen. Mit dieser zweiten Frage möchte ich mich im Folgenden auseinandersetzen.

\section{Für wirtschaftliche Interessen in den Krieg ziehen?}

Weil ich diese Frage aus der Perspektive der evangelischen Sozialethik analysieren werde, greife ich zurück auf eine klassische Kontroverse in der lutherischen Orthodoxie: den interimistischen oder adiaphoristischen Streit. Während Philipp Melanchthon einen Kompromiss mit der katholischen Kirche suchte und daher im Leipziger Interim 1548 bei Nebensächlichkeiten Zugeständnisse einräumte, um einen Krieg zu vermeiden, protestierte der strenge Lutheraner Matthias Flacius Illyricus aus Magdeburg gegen diese Nachgiebigkeit und betonte, dass es im Bekenntnisfall (status confessionis) keine Nebensächlichkeiten geben dürfe und man standhaft bleiben müsse, weil sonst eine Selbstaufgabe drohe ${ }^{39}$. Kontrovers war demzufolge, ob es solche Adiaphora (Nebensächlichkeiten) gibt, oder ob nicht vielmehr jedes Thema in einem Konfliktfall zu einer Grundsatzfrage werden kann, bei der man keinesfalls weichen darf. Diese Streitfrage wurde nicht rechtlich, sondern aus der theologischethischen Perspektive der betroffenen Akteure diskutiert. Als ethische Frage formuliert lautet sie: Welchen Stellenwert hat ein politischer Kompromiss aus evangelischer Perspektive und welche Themen oder Positionen sind nicht verhandelbar? ${ }^{40}$

Analog kann heute gefragt werden, ob wirtschaftliche Interessen so gravierend sein können, dass sie sogar den Einsatz des Militärs rechtfertigen oder ob sie nicht vielmehr zu den Nebensächlichkeiten gehören, für die man sich zwar engagieren darf und soll, die aber niemals Gewaltanwendung erlauben. Diese Frage darf natürlich den gegenwärtigen völkerrechtlichen Rahmen nicht vernachlässigen: Nach der Charta der Vereinten Nationen von 1945 herrscht in den internationalen Beziehungen ein explizites Gewaltverbot (Art. 2.4), sodass Kriege (als typischer Fall einer Androhung und Anwendung von staatlicher Gewalt) verboten

38 Die Schutzverantwortung besteht nicht nur aus der humanitären Intervention (responsibility to react), sondern umfasst auch und zuerst präventive Maßnahmen (responsibility to prevent) sowie die Hilfe zum Wiederaufbau (responsibility to rebuild). Zudem ist die Androhung und Ausübung militärischer Gewalt selbst innerhalb der reaktiven Maßnahmen nur eine Option, der friedliche Maßnahmen (Diplomatie, Schiedssprüche, Boykott) vorangehen sollten. Nur in vier Fällen ist gemäß der UN-Resolution 60/1 von 2005 militärische Gewalt denkbar: Völkermord, schwerste Menschenrechtsverletzungen, Kriegsverbrechen, ethnische Säuberungen. Vgl. dazu Christopher Verlage, Responsibility to Protect. Ein neuer Ansatz im Völkerrecht zur Verhinderung von Völkermord, Kriegsverbrechen und Verbrechen gegen die Menschlichkeit, Tübingen 2009.

39 Vgl. das Handbuch der Dogmen- und Theologiegeschichte Band 2: Die Lehrentwicklung im Rahmen der Konfessionalität, hrsg. von Carl Andresen und Adolf Martin Ritter, 2. Auflage Göttingen 1998, 109-113.

40 Vgl. dazu aktuell Avishai Margalit, Über Kompromisse - und faule Kompromisse, Berlin 2011. Er räumt den Kompromiss einen großen Spielraum ein, weil und sofern Kompromisse friedensförderlich sind. Der Friede hat für Margalit also Priorität gegenüber der Gerechtigkeit (vgl. 95ff.). Die Grenze wird jedoch erreicht, wenn Menschen erniedrigt und verachtet werden. Dabei rekurriert auch Margalit auf eine theologische Argumentationsfigur, indem er ein religiöses Politikverständnis von einem ökonomischen absetzt (vgl. 28ff.): Während im Medium des Geldes alles vergleichbar und damit verhandelbar sei, kenne die Religion das Tabu, das nicht verhandelbar ist. 
$\operatorname{sind}^{41}$. Vielmehr verpflichten sich alle Mitglieder dazu, Streitigkeiten mit friedlichen Mitteln beizulegen (Art. 2.3). Lediglich das Recht auf Selbstverteidigung wird zugestanden (Art. 51), aber auch diese militärische Gewalt wird doppelt eingehegt, indem der Angegriffene seine Maßnahmen dem Sicherheitsrat anzuzeigen hat und sie beenden muss, sobald der Sicherheitsrat die erforderlichen Maßnahmen getroffen hat. Darüber hinaus können (als Ultima Ratio) humanitäre Interventionen vom Sicherheitsrat beschlossen werden (Kapitel VII). Es gibt also kein freies Kriegführungsrecht der Völker bzw. Herrscher mehr (wie zu Zeiten der Reformatoren), sondern ein System kollektiver Sicherheit, das einen Krieg aus wirtschaftlichen Interessen nicht dulden kann und darf. „Kurz: Wirtschaftskriege sind rechtlich nicht zulässig“42.

Jedoch gibt es zum einen interne Spannungen in der Konzeption der Vereinten Nationen ${ }^{43}$, zum anderen externe Veränderungen gegenüber 1945 (wie Globalisierung, Migration, Staatenzusammenbrüche und neue Kriege ${ }^{44}$ ), sodass inzwischen diskutiert wird, inwieweit Anpassungsbedarf der Ausführungen der UNCharta bestehe - sei es des Wortlauts, sei es der Auslegungspraxis. Wer angesichts dieser Spannungen davon überzeugt ist, dass Militäreinsätze aus wirtschaftlichen Interessen weiterhin nicht sein dürfen, sollte ethisch argumentieren, um das Gewaltverbot der UN-Charta zu untermauern - und dazu greife ich auf den adiaphoristischen Streit und seine Argumente zurück, den ich nunmehr nicht völkerrechtlich, sondern mit Blick auf mögliche politische Entwicklungen beurteile.

Die Pointe im adiaphoristischen Streit liegt darin, dass beide Positionen etwas Richtiges betont haben. Flacius ist darin zuzustimmen, dass man nicht vorab Konfliktfälle ausschließen oder verharmlosen kann. Im Bekenntnisfall, wenn es also um das Ganze geht - und das war damals die evangelische Wahrheit über Gott und Jesus Christus ${ }^{45}$ - wäre ein Nachgeben von einer Selbstaufgabe nicht zu unterscheiden. Wer solche vermeintlichen Nebensächlichkeiten vorab festlegt, hat sie schon aufgegeben. Mit Blick auf wirtschaftliche Interessen ist es dementsprechend illusorisch, bestimmte Interessen vorab als beliebig einzustufen. Bereits in den 1970er Jahren wurde

41 In der Präambel bekennen die „Völker der Vereinten Nationen“, dass sie fest entschlossen seien, „künftige Geschlechter vor der Geißel des Krieges zu bewahren, die zweimal zu unseren Lebzeiten unsagbares Leid über die Menschheit gebracht hat" - zitiert nach: Charta der Vereinten Nationen und das Statut des Internationalen Gerichtshofs, hrsg. von Hartmut Krüger, Stuttgart 1995, 11.

42 Franz C. Mayer, Außenpolitische Interessen aus rechtlicher Sicht - blinde Flecken des Verfassungsrechts?, in: Was sind legitime außenpolitische Interessen? Unverfügbare Voraussetzungen des säkularen Staates. Umgang mit Schuld in der Öffentlichkeit. Werner-Reihlen-Vorlesungen 2010 bis 2012 , hrsg. von Notger Slenczka, Leipzig 2013, 12-32, 24.

43 Mit Blick auf die Erlaubnisregelung zur Selbstverteidigung ist für dieses Thema vor allem die Frage relevant, was erforderliche Maßnahmen des Sicherheitsrates sind, die das Verteidigungsrecht nach Art. 51 limitieren. Mit Blick auf das Gewaltverbot wird angefragt, ob es nicht eine Schutzverantwortung oder schützenswerte Güter (wie die Menschenrechte) gibt, die eine humanitäre Intervention gebieten können, die dann nicht gegen das Gewaltverbot der UN-Charta verstießen. Schließlich gibt es das Problem, dass der Sicherheitsrat keine eigenen Streitkräfte befehligt - und bei der Delegation an Mitgliedstaaten können immer auch deren Partikularinteressen eine Rolle spielen.

44 Vgl. beispielsweise Shannon D. Beebe und Mary Kaldor, Unsere beste Waffe ist keine Waffe. Konfliktlösungen für das 21. Jahrhundert, Berlin 2012, 13 54.

45 Zur Verbindung zwischen dem Beharren auf nicht aufgebbaren Wahrheiten und dem religiösen Fundamentalismus vgl. Karen Armstrong, Im Kampf für Gott. Fundamentalismus in Christentum, Judentum und Islam, München 2007. bemerkt, dass unser Wohlstand insbesondere von Ölimporten abhängig ist, sodass das Sicherheitsverständnis um den ökonomischen Bereich erweitert wurde. Dennoch stand ein Krieg um sauberes Wasser damals wahrscheinlich noch nicht im Fokus. Inzwischen können wir uns ohne weiteres vorstellen, dass ein zur Bewässerung des Ackerlandes oder zur Kühlung von Industrieanlagen gestauter Oberlauf die elementare wirtschaftliche Versorgung bei den weiteren Anliegern des Flusses gefährden kann. Ebenso könnten das Leerfischen ganzer Meeresgebiete oder die Verschmutzung einer Region zu einer Gefahr für die wirtschaftliche Grundversorgung eines Landes anwachsen. Sofern Horst Köhler mit seinem Verweis auf einen Notfall solche Szenare im Blick hatte, brachte er etwas Bedenkenswertes zur Sprache. Allerdings muss man darauf achten, dass hier nicht Notfälle, welche die menschliche Sicherheit gefährden (wie das Verdursten eines Volkes), zu wirtschaftlichen Interessen erklärt werden ${ }^{46}$ !

Aber es gibt auch eine andere Seite der Medaille: Um einen Krieg zu vermeiden, reicht es nicht, nur rhetorisch auf Diplomatie und Gespräche zu setzen, man muss auch inhaltlich bereit sein, Kompromisse einzugehen, und das heißt eben auch, in manchen Positionen nachzugeben und sich wechselseitig anzunähern. Und welche Positionen das sein können, sollte durchaus vorab in der eigenen Partei diskutiert werden - das ist die kluge Einsicht des Wittenberger Reformators Melanchthon. Beratungsresistenz und das Beharren auf Maximalpositionen - und die werden ja bezogen, wenn man auf die evangelische Wahrheit oder politisch auf die freiheitlich demokratische Grundordnung rekurriert verhindern eine Annäherung und verhärten die Fronten. Solche Bockigkeit könnte sogar einen Krieg herbeiführen, der wohl mehr zerstört, als strittig war. Im angedachten Szenar würden ein zerwühltes Ackerland und ein vergifteter Fluss, der den ungefilterten Abfall der zerstörten Industrieanlagen transportiert, beiden Ländern und ihren Bewohnern schaden. Aus dieser Perspektive war die Äußerung des damaligen Bundespräsidenten unpassend, weil sie unnötig eskalierte.

Als Zwischenergebnis lässt sich demnach festhalten, dass ein Militäreinsatz aus wirtschaftlichen Interessen zwar leider nicht apriorisch und für alle Fälle auszuschließen ist, dass es aber diplomatisch ungeschickt sein und als militärische Drohung aufgefasst werden kann, wenn man ohne konkreten Anlass dieses Szenario heraufbeschwört.

Nunmehr sollen die Erkenntnisse aus dem veränderten Sicherheitsdiskurs mit einbezogen werden. Dadurch wird sich die Kritik an einem militärischen Gewalteinsatz aus wirtschaftlichen Gründen noch verschärfen. Durch die Kopenhagener Brille betrachtet erscheint die Rede von einer militärischen Sicherung oder gar Durchsetzung wirtschaftlicher Interessen als der Versuch, die Risiken einer durchgehend globalisierten Weltwirtschaft erstens als sicherheitspolitisch äußerst relevant einzustufen und zweitens das Militär als kompetenten Player ins Spiel zu bringen. Beide Annahmen werden nunmehr analysiert:

46 Die entsprechende Argumentation würde im angesprochenen Beispiel lauten: Wenn das Verdursten eines Volkes notfalls mit militärischer Gewalt verhindert oder gestoppt werden dürfe und wenn die Verhinderung eines solchen Verdurstens ein wirtschaftliches Interesse (und eben nicht ein Aspekt menschlicher Sicherheit) sei, dann wäre der Einsatz militärischer Gewalt für wirtschaftliche Interessen legitimierbar. 
(1) Dass wirtschaftliche Interessen ethisch neutral und politisch relevant sind, wurde bereits festgehalten. Der Vorwurf gegen die Kopenhagener Schule, hier würden nur Szenare konstruiert, ohne zu bedenken, ob sie auch realen Anhalt haben ${ }^{47}$, läuft ins Leere. Das Problem liegt nicht darin, dass die genannten Risiken nur im Kopf (bzw. im Reden) ihrer Protagonisten existieren, sondern darin, dass es gleichsam mehr Bewerber als Stellen gibt: Es gibt sehr viele Risiken, aber nicht alle werden gleichermaßen politisch berücksichtigt werden können. Damit ein Risiko als sicherheitspolitisch relevant anerkannt wird, müssen also zwei Hürden genommen werden: Das Risiko muss erstens real sein und zweitens müssen die politischen Akteure von dessen inhärenter Gefährlichkeit überzeugt werden. Ich behaupte, dass beide Bedingungen von dem Risikofaktor der wirtschaftlichen Interessen erfüllt worden sind. Die Weltwirtschaft ist mit sicherheitspolitisch relevanten Risiken verbunden und diese Risiken sind von der Politik auch erkannt und anerkannt. Allerdings wird hierbei eindeutig auf den erweiterten Sicherheitsbegriff rekurriert, denn der Raum ist die globale vernetzte Welt, der Sache nach geht es um ökonomische Bedrohungen, gemäß der Gefahrendimension handelt es sich um ein Risiko und geschützt werden soll die Gesellschaft. Bereits diese Beobachtung lässt die Vermutung aufkommen, dass nicht nur und nicht einmal vorrangig der Staat und seine Exekutive an dieser Stelle für Sicherheit sorgen können.

(2) Dass militärische Kräfte wirtschaftliche Interessen verteidigen oder schützen können, ist weitaus weniger schlüssig - vor allem, wenn weiterhin bedacht wird, was erstens mit dem Begriff der wirtschaftlichen Interessen alles gemeint ist und dass er zweitens nicht mit der Gefährdung menschlicher Sicherheit identifiziert werden darf. Einige provokative Fragen zur Verdeutlichung:

- Soll die Bundeswehr einschreiten, wenn ein Land seine Güter nicht an ein Unternehmen aus Deutschland verkaufen möchte? Und wer sollte bekämpft werden, das nicht verkaufsbereite Land oder dasjenige Land, das stattdessen den Zuschlag erhielt?

- Wenn zwar der Verkauf der Güter klappt, nicht aber der Transport, ist dann das Militär oder der Spediteur zuständig? Würden militärische Maßnahmen nicht den freien Handel unterlaufen, weil bestimmte Unternehmen durch den Staat bevorzugt würden? Könnten diese Unternehmer dann nicht Kosten externalisieren, die der Staat bei ihnen, nicht aber bei ihren Konkurrenten übernähme?

- Wenn andere Länder nicht an unseren Wirtschaftsgütern interessiert sind, weil sie kaum wirtschaftliche Interessen haben, darf die Bundeswehr dann mit Gewalt nachhelfen, damit wir Exportweltmeister bleiben?

- Wenn ein Land oder Unternehmen in diesem Land Arbeiter ausbeuten, um möglichst preiswert Sportstätten aufzubauen, also die menschliche Sicherheit der Arbeiter aus wirtschaft-

47 Vgl. Jörg Friedrichs, Sinn und Zweck der Nachhaltigkeitstransformation: Von ontologischer Unsicherheit zu sozioökologischer Resilienz; in: Verunsicherte Gesellschaft - überforderter Staat. Zum Wandel der Sicherheitskultur, hrsg. von Christopher Daase et al., Frankfurt/Main 2013, 259-276. lichem Interesse gefährden, wäre dann eine humanitäre Intervention gerechtfertigt?

Diese Fragen sollen aufzeigen, in welche Schieflage unsere Exekutive geriete ${ }^{48}$. Grundlegend gilt: Wenn bestimmte Güter für Deutschland sehr wichtig wären, so müsste deutsche Politik in der Tat proaktiv eingreifen, um das eigene wirtschaftliche Interesse zu sichern und das korrespondierende Risiko zu entschärfen - aber nicht militärisch, sondern zunächst mit wirtschaftlichen Maßnahmen, bspw. indem das deutsche Unternehmen subventioniert wird, damit es dem potenziellen Anbieter einen überzeugenden Preis bieten kann. Des Weiteren wäre denkbar, dass die deutsche Politik wissenschaftliche Forschungen unterstützt, um Alternativen zu dieser Güterabhängigkeit zu entwickeln. Und natürlich gibt es auch andere politische Mittel wie Diplomatie, um hier zu einer akzeptablen Lösung zu kommen; so dürfte die Androhung eines Boykotts der Sportereignisse weitaus effektiver sein als die Androhung militärischer Reaktionen. Zudem sollte Deutschland darauf reflektieren, inwiefern es selbst durch seine Politik nicht nur Opfer und Helfer, sondern auch Mitverursacher oder Verstärker solcher Risiken ist ${ }^{49}$, um nachhaltige Lösungsstrategien verfolgen zu können.

Wer hingegen behauptet, dass Deutschland seine durchaus berechtigten wirtschaftlichen Interessen gegebenenfalls mithilfe des Militärs durchsetzen müsste, begeht den Fehler, dass er die neuen sicherheitspolitischen Herausforderungen mit den alten Mitteln meint meistern zu können ${ }^{50}$. Er macht aus wirtschaftlichen Konkurrenten politische Feinde. „Smart power" (Joseph Nye ${ }^{51}$ ) sieht anders aus! Damit leugne ich weder, dass wirtschaftliche Interessen verfolgt werden dürfen, noch spreche ich der Bundeswehr ihr Existenzrecht ab, noch bestreite ich, dass es legale und legitime Einsätze der Bundeswehr geben kann, nur bezweifle ich, dass militärische Gewalt in diesem Szenar hilfreich sein kann. Militärische Gewalt ist ein untaugliches Mittel, um wirtschaftliche Probleme zu lösen - und weil die Frage nach der Geeignetheit und Verhältnismäßigkeit der Mittel zum Analysepotenzial einer politischen Ethik zählen,

48 Das aktuelle „Bananenvideo“ der Marine, das insinuiert, die Marine werde dazu eingesetzt, den Export von Bananen sicherzustellen und müsse darum Piraten bekämpfen, ist ein lehrreiches Beispiel für eine solche argumentative Schieflage. Wenn die Beteiligung an der Operation Atalanta vor der Küste Somalias nicht dazu diente, die Hilfsleistungen des World-Food-Programms sicher zu eskortieren und Geiselnahmen durch Piraten zu verhindern, also Verbrechen zu verhindern, sondern dazu, unsere Versorgung mit preiswerten Bananen zu gewährleisten, dann wäre dies eine äußerst bedenkliche Aussage. Grundsätzliche Erwägungen zur problematischen Legitimität dieses Militäreinsatzes finden sich bei Volker Stümke, Überlegungen zur Legitimität der Operation Atalanta; in: Zeitschrift für evangelische Ethik 54, Gütersloh 2010, 47-57.

49 Vgl. Sabine Jaberg, Vernetzte Sicherheit? Phänomenologische Rekonstruktion und kritische Reflexion eines Zentralbegriffs im Weißbuch 2006, HSW kontrovers 5, Hamburg 2009

50 Die Behauptung, dass eine solche Redeweise die Wahrscheinlichkeit eines realen Krieges aus wirtschaftlichen Interessen erhöhen kann, kann sich im Analogieschluss berufen auf die Dissertation von Markus Kink, Die Sprache des Krieges. Zur diskursiven Ermöglichung präventiver Kriegsführung, Baden-Baden 2011. Kink arbeitet heraus, dass und wie die Rede vom Krieg als Deutungskategorie in den Anfangsjahren des Kalten Krieges sowie in den Debatten um den Irakkrieg den sicherheitspolitischen Diskurs beeinflusste und 2003 sogar das Führen eines präemptiven Krieges ermöglichte.

51 Vgl. Joseph S. Nye, Macht im 21. Jahrhundert. Politische Strategien für ein neues Zeitalter, München 2011 
ist dieser Befund ein ethisch relevantes Ergebnis ${ }^{52}$. Wirtschaftskriege sind also nicht nur rechtlich verboten, sie sind auch aus ethischer Perspektive abzulehnen.

52 Zumindest hinzuweisen ist auf die Gefahr, dass eine solche Argumentation die Privatisierung und Finanzialisierung der Gewalt unterstützen könnte (vgl. dazu Erhard Eppler, Vom Gewaltmonopol zum Gewaltmarkt? Die Privatisierung und Kommerzialisierung der Gewalt, Frankfurt/Main 2002). Private Gewaltunternehmer könnten sich als Alternative zum staatlichen Gewaltmonopol profilieren und damit könnte Sicherheit von der Finanzkraft privater Akteure abhängig werden. Mit dieser Entwicklung würde das wirtschaftliche Paradigma über die Politik dominieren. Daher bleibt es Aufgabe der Politik, ihr Gewaltmonopol nicht aushöhlen zu lassen - bspw. durch klare Rahmenbedingungen. Und an wirtschaftliche Unternehmen müsste die Frage gestellt werden, ob sie sich nicht dieser Entwicklung selbst entgegenstellen sollten, denn eine Schwächung des staatlichen Gewaltmonopols würde in der Folge auch die Unternehmen treffen.

\title{
Europäische Migrations- und Außengrenzpolitik - Wege aus der Sackgasse?
}

\section{Ruth Vollmer*}

\begin{abstract}
Increasingly challenged by a growing number of deaths in the Mediterranean and critical public debates, EU policy makers continue their more-of-the-same approach regarding external border policies, turning to the prevailing security-focussed paradigm for a solution. This paper argues that such an approach will do nothing to solve the problem of deadly and inhumane external borders, but may well exacerbate it. Although conceptions of migration vary (development potential, demographic necessity, security risk), the European migration and border regime appears to have developed a self amplifying dynamic, while real alternatives seem politically blocked. This article argues that central assumptions underlying the current approach do not hold and suggests policy alternatives, which would strengthen coherence between the EU's border policies and human rights agenda taking into account inherent tensions between migration and the (welfare) state.
\end{abstract}

Keywords: EU, borders, migration, externalizaton;

EU, Grenzen, Migration, Externalisierung

\section{Einleitung}

Am 3. Oktober 2013 starben weniger als eine Meile vor der Küste Lampedusas mehr als 360 Menschen aus Eritrea und Somalia, nachdem ihr Boot mit insgesamt ca. 500 Menschen an Bord nach 13 Tagen auf See in Brand geraten war und sank. Eines von drei Fischerbooten, die sich während des Brandes in Sichtweite des Schiffes aufhielten, leistete Hilfe und konnte 47 der Flüchtlinge retten, jedoch erst nachdem deren Boot bereits gekippt war und alarmierte auch erst dann die Küstenwache, die 45 Minuten später eintraf. Die Geretteten wurden zu einer Geldstrafe wegen „illegaler Einwanderung“ verurteilt und zunächst im Auffanglager in Lampedusa, das damals etwa viermal so viele Personen beherbergte, wie vorgesehen, interniert; die Verstorbenen erhielten ein Staatsbegräbnis. ${ }^{1}$

* $\quad$ Ruth Vollmer hat 2008 ihr Magisterstudium der Politikwissenschaft, Sprachwissenschaft und Psychologie an der Universität Bonn abgeschlossen; sie arbeitet derzeit am Internationalen Konversionszentrum Bonn (BICC). Dieser Artikel wurde einem anonymisierten Begutachtungsverfahren unterzogen (double-blind peer-reviewed). Die Autorin bedankt sich bei den Gutachtern für wertvolle Hinweise und hilfreiche Kommentare.

1 Perkowski, Nina (2013): No accident. http://opendemocracy.net/5050/ nina-perkowski/no-accident-why-have-19142-died-at-europe\%E2\%80\%99sfrontiers (eingesehen am 17.1.14)
Lampedusa, eine kleine italienische Mittelmeerinsel mit ca. 5.000 Einwohnern, die geografisch näher an Tunesien als an Italien liegt, ist seither umso mehr zu einem Synonym für das Sterben von Menschen an Europas Außengrenzen geworden. Denn anders als unzählige vorhergehende ähnliche Vorfälle ${ }^{2}$ sorgte diese „Tragödie“, möglicherweise aufgrund ihrer Nähe zu Europa und der vielen Toten, für ein außergewöhnlich starkes Medienecho und einen „unprecedented call for action by EU leaders and citizens, “3 der darauf zielte, Ähnliches in Zukunft zu verhindern.

Zwei Monate nach „Lampedusa“ legte die Task Force for the Mediterranean ihren Bericht vor, den die zuständige EUKommissarin als eine "truly European response that can make a difference ${ }^{\prime 4}$ ankündigte. Darin wird ein integrierter Ansatz

2 Die Internationale Organisation für Migration schätzt, dass seit 199320.000 Menschen an den europäischen Außengrenzen ums Leben gekommen sind. Solche Zahlen stehen für eine vermutlich deutlich höhere Dunkelziffer. http:// www.iom.int/cms/render/live/en/sites/iom/home/news-and-views/pressbriefing-notes/pbn-2013/pbn-listing/iom-mourns-lampedusa-shipwreck-v. html (eingesehen am 17.1.14).

3 European Commission (2013): Communication from the European Commission the European Parliament and the Council on the work of the Task Force Mediterranean. COM(2013) 869 final. Brüssel.

4 Malmström, Cecilia (2013): Preventing deaths in the Mediterranean. http://ec.europa.eu/commission_2010-2014/malmstrom/news/ archives/2013/12/20131204_en.htm (eingesehen am 17.1.14). 